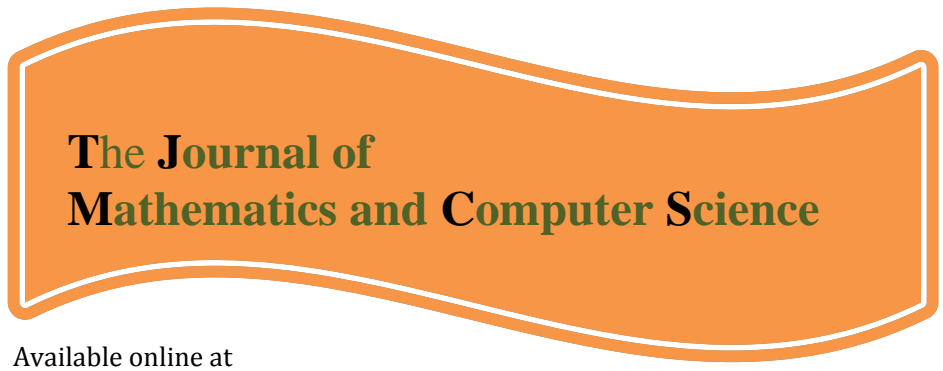

http://www.TJMCS.com

The Journal of Mathematics and Computer Science Vol .2 No.2 (2011) 295-306

\title{
Comparison of two methods for solving fuzzy differential equations based on Euler method and Zadeh's extension
}

\section{H. Saberi Najafi ,F. Ramezani Sasemasi,S. Sabouri Roudkoli, S. Fazeli Nodehi}

\author{
Department of Mathematics, Faculty of Sciences, Islamic Azad university of Lahijan \\ Department of Mathematics, Faculty of Sciences, Guilan University, P.O. Box 41335-1914, Rasht, Iran \\ Computer Center, Guilan University, Rasht, Iran, hnajafi@guilan.ac.ir \\ Department of Mathematics, Islamic Azad university of Lahijan ramezani_fateme09@yahoo.com \\ Department of Mathematics, Islamic Azad university of Lahijan sara_smart22@yahoo.com \\ Department of Mathematics, Islamic Azad university of Lahijan saeide_fazeli@yahoo.com
}

Received: July 2010, Revised: October 2010

Online Publication: January 2011

\begin{abstract}
In this paper, two important methods which apply for solving Fuzzy differential equations are compared. These methods are:

1. Zadeh extension principal

2. Standard Euler method

The methods are compared by numerical examples.Also in each case by approximating the errors, the converges of the methods will be considered.

The results are shown in tables and figures.
\end{abstract}

Keywords: Fuzzy differential equations, Zadeh's extension, Euler method.

\section{Introduction}

In 1972, Chang and Zadeh [27] first introduced the concept of fuzzy derivative, followed up ten years later by Dubois and Prade [28], who used the extension principle in their approach. 
This paper is organized as follows: in Section 2, we recall some basic definitions and theoretical background that we need throughout this paper.

In section 3 we define the problem, this is a fuzzy Cauchy problem [11,9] whose numerical solution is the main interest of this work and we apply the standard Euler method for systems $[1,7,8,13]$ followed by a complete error analysis and show that the numerical solution converges to the unique solution.

In section 4, we study FDE where the fuzzy function is obtained via Zadeh's extension principle. We get a fuzzy solution for this class of FDE[2,4].

In section 5, the mentioned method has been applied to two examples, finally we compare these two methods with each other.

\section{Preliminaries}

In this following the most basic notations used in fuzzy calculus are introduced. We start by defining the fuzzy number.

\section{Definition 2.1}

A fuzzy number is a fuzzy set $\mathrm{u}: \mathrm{R}^{1} \rightarrow \mathrm{I}=[0,1]$ which satisfies

i. $\mathrm{u}$ is upper semicontinuous.

ii. $\mathrm{u}(\mathrm{x})=0$ outside some interval $[\mathrm{c}, \mathrm{d}]$.

iii. There are real numbers $\mathrm{a}, \mathrm{b}: \quad \mathrm{c} \leq \mathrm{a} \leq \mathrm{b} \leq \mathrm{d}$ for which

1. $\mathrm{u}(\mathrm{x})$ is monotonic increasing on $[\mathrm{c}, \mathrm{a}]$,

2. $u(x)$ is monotonic decreasing on $[b, d]$,

3.u(x)=1, $\mathrm{a} \leq \mathrm{x} \leq \mathrm{b}$

The set of all the fuzzy numbers (as given by Definition 2.1) is denoted by $\mathrm{E}^{1}$. An alternative definition which yields the same $\mathrm{E}^{1}$ is given by Kaleva [21].

\section{Definition 2.2}

Let $U$ be a fuzzy set on $R$ [4]. $U$ is called a fuzzy interval if:

(i) $U$ is normal: there exists $x_{0} \in R$ such that $U\left(x_{0}\right)=1$;

(ii) $U$ is convex: for all $x, t \in R$ and $0 \leq \lambda \leq 1$, it holds that

$$
\mathrm{U}(\lambda \mathrm{x}+(1-\lambda) \mathrm{t}) \geq \min (\mathrm{U}(\mathrm{x}), \mathrm{U}(\mathrm{t}))
$$

(iii) $\mathrm{U}$ is upper semi-continuous: for any $\mathrm{x}_{0} \in \mathrm{R}$, it holds that

$$
\mathrm{U}\left(\mathrm{x}_{0}\right) \geq \lim _{\mathrm{x} \rightarrow \mathrm{x}} \pm \mathrm{U}(\mathrm{x})
$$

(iv) $[\mathrm{U}]^{0}=\mathrm{CL}\{\mathrm{x} \in \mathrm{R}$ : $\mathrm{U}(\mathrm{x})>0\}$ is a compact subset of $\mathrm{R}$.

The $\alpha$-cut of a fuzzy interval $U$, with $0<\alpha \leq 1$ is the crisp set

$$
[\mathrm{U}]^{0}=\{\mathrm{x} \in \mathrm{R} \vdots \mathrm{U}(\mathrm{x})>0\} .
$$

For a fuzzy interval $U$, its $\alpha$-cuts are closed intervals in $R$. Let denote them by

$$
[\mathrm{U}]^{0}=\left[\mathrm{u}_{1}^{\alpha}, \mathrm{u}_{2}^{\alpha}\right] \text {. }
$$

If $u \in F(R)$, then $u$ is called a fuzzy interval and the $\alpha$-level set $[u]^{\alpha}$ is a nonempty compact interval for all $\alpha \in[0,1]$.

Let $\quad u, v \in E^{n}$ and let $c$ be a positive number. The addition $u+v$ and (positive) scalar

Multiplication $c . u$ in $E^{n}$ are defined in terms of the $a$-level sets by

$[u+v]^{\alpha}=[u]^{\alpha}+[v]^{\alpha}, \quad[c . u]^{\alpha}=c[u]^{\alpha}$,

for every $\alpha \in[0,1]$ 


\section{Definition 2.3}

A fuzzy interval $U$ is called a triangular fuzzy interval if its membership function has the following form:

$$
U(x)=\left\{\begin{array}{cc}
0 & \text { if } x<a \\
\frac{x-a}{b-a} & \text { if } a \leq x \leq b \\
\frac{c-x}{c-b} & \text { if } b \leq x \leq c \\
0 & \text { if } x>c
\end{array}\right.
$$

and its $\alpha$ - cuts are simply

$[U]^{\alpha}=[a+\alpha(b-a), c-\alpha(c-b), \alpha \in(0,1]][5]$.

In this paper, the set of all fuzzy intervals is denoted by $F(R)$.

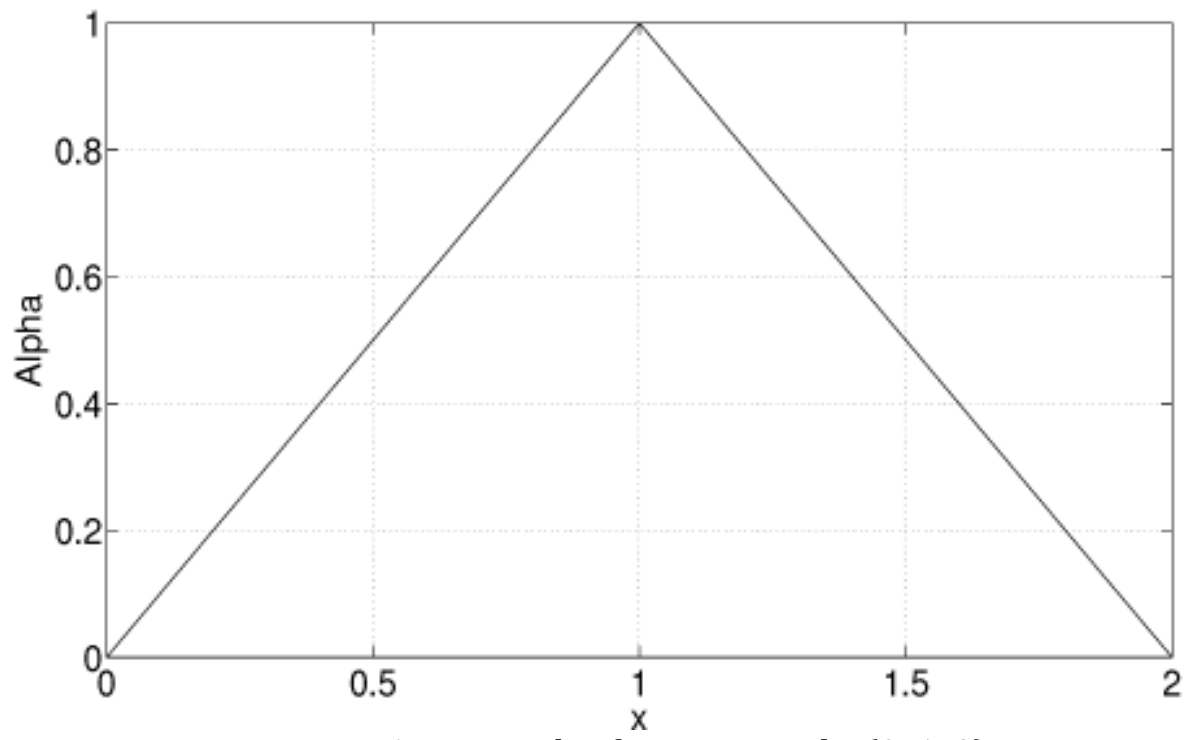

Figure 1: Triangular fuzzy interval $\mathrm{U}(0,1,2)$.

We denote by $(a, b, c)$ the triangular fuzzy number with support [a, c].

We will next define a metric $\mathrm{D}$ in $\mathrm{E}^{1}$ and the fuzzy function notation [20].

\section{Definition 2.4}

For arbitrary fuzzy numbers $u=(\underline{u}, \bar{u})$ and $\mathrm{v}=(\underline{\mathrm{v}}, \overline{\mathrm{v}})$ the quantity

$\mathrm{D}(\mathrm{u}, \mathrm{v})=\sup _{0 \leq \alpha \leq 1} \quad\{\max [|\underline{\mathrm{u}}(\alpha), \underline{\mathrm{v}}(\alpha)|,|\overline{\mathrm{u}}(\alpha), \overline{\mathrm{v}}(\alpha)|]\}$ 
is the distance between $\mathrm{u}$ and $\mathrm{v}$.

This metric is equivalent to the one used by Purl and Ralescu [23] and Kaleva [21]. It is shown [24] that $\left(\mathrm{E}^{1}, \mathrm{D}\right)$ is a complete metric space.

This fuzzy number space as shown in [16], can be embedded into the Banach space $\mathrm{B}=\bar{C}[0,1] \times \bar{C}[0,1]$ where the metric is usually defined as

$\|(\mathrm{u}, \mathrm{v})\|=\max \left\{\sup _{0 \leq \alpha \leq 1}|\mathrm{u}(\alpha)|, \sup _{0 \leq \alpha \leq 1}|\mathrm{v}(\alpha)|\right\}$

for arbitrary $(\mathrm{u}, \mathrm{v}) \in \bar{C}[0.1] \times \bar{C}[0.1]$. Throughout this work we will use the sup norm given by Eq. (4) rather than the $L_{p}$ norm [12.15]. Using this norm enables to obtain stronger results related to the numerical procedure.

Also, we can extend the Housdorff metric $\mathrm{H}$ on

$D: \mathrm{R}_{\mathrm{F}} \times R_{F} \rightarrow R_{+} \cup\{0\}$ by means of

$\mathrm{D}(\mathrm{u}, \mathrm{v})=\sup _{\alpha \in[0,1]} \max \left\{\underline{\mathrm{u}^{\alpha}}-\underline{\mathrm{v}^{\alpha}}|,| \overline{\mathrm{u}^{\alpha}}-\overline{\mathrm{v}^{\alpha}} \mid\right\} \quad, \forall \mathrm{u}, \mathrm{v} \in \mathrm{R}_{\mathrm{F}}$

(see [10]).

\section{Definition 2.5}

A function $\mathrm{f}: \mathrm{R}^{1} \rightarrow \mathrm{E}^{1}$ is called a fuzzy function. If for arbitrary fixed $\mathrm{t}_{0} \in \mathrm{R}^{1}$ and $\varepsilon>0$, a $\delta>0$ such that

$\left|\mathrm{t}-\mathrm{t}_{0}\right|<\delta \Rightarrow \mathrm{D}\left[\mathrm{f}(\mathrm{t}), \mathrm{f}\left(\mathrm{t}_{0}\right)\right]<\varepsilon$

exists, $\mathrm{f}$ is said to be continuous.

Throughout this work we also consider fuzzy functions which are defined only over a finite interval [a, b] (we simply replace $\mathrm{R}^{1}$ by [a, b] in Definition 2.5).

\section{Definition 2.6}

Let $u, v \in F\left(R^{n}\right)$ be. If there exists $\mathrm{w} \in \mathrm{F}\left(\mathrm{R}^{\mathrm{n}}\right)$ such that

$u=v+w$ then $\mathrm{w}$ is called the H-difference of $\mathrm{u}$ and $\mathrm{v}$ and it is denoted by $\mathrm{u}-\mathrm{v}$.

\section{Definition 2.7}

Let $\quad \mathrm{f}: \mathrm{T} \rightarrow \mathrm{F}\left(\mathrm{R}^{\mathrm{n}}\right)$ be and $t_{0} \in \mathrm{T}$.We say that $\mathrm{x}$ is differentiable at $\mathrm{t}_{0}$ if:

(I) There exists an element $f^{\prime}\left(t_{0}\right) \in F\left(R^{n}\right)$ such that, for all $\mathrm{h}>0$ sufficiently near to 0 , there are $\mathrm{f}\left(\mathrm{t}_{0}+\mathrm{h}\right)-\mathrm{f}\left(\mathrm{t}_{0}\right), \mathrm{f}\left(\mathrm{t}_{0}\right)-\mathrm{f}\left(\mathrm{t}_{0}-\mathrm{h}\right)$ and the limits (in D-metric)

$f^{\prime}\left(t_{0}\right)=\lim _{h \rightarrow 0^{+}} \frac{f\left(t_{0}+h\right)-f\left(t_{0}\right)}{h}=\lim _{h \rightarrow 0^{+}} \frac{f\left(t_{0}\right)-f\left(t_{0}-h\right)}{h}$

Or

(II) There is an element $\mathrm{f}^{\prime}\left(\mathrm{t}_{0}\right) \in \mathrm{F}\left(\mathrm{R}^{\mathrm{n}}\right)$ such that,for all $\mathrm{h}<0$ sufficiently near to 0 , there are $f\left(t_{0}+h\right)-f\left(t_{0}\right), f\left(t_{0}\right)-f\left(t_{0}-h\right)$ and the limits

$f^{\prime}\left(t_{0}\right)=\lim _{h \rightarrow 0^{-}} \frac{f\left(t_{0}+h\right)-f\left(t_{0}\right)}{h}=\lim _{h \rightarrow 0^{-}} \frac{f\left(t_{0}\right)-f\left(t_{0}-h\right)}{h}$

Note that derivative in the first form (I) is coincident with the $\mathrm{H}$-derivative .Also, if $\mathrm{x}$ is differentiable in the first form (I) and $\mathrm{f}^{\prime}\left(\mathrm{t}_{0}\right) \in \mathrm{F}\left(\mathrm{R}^{\mathrm{n}} / \mathrm{R}^{\mathrm{n}}\right)$ then, from Definition 2.7, it is not differentiable in the second form (II) and vice versa.

\section{Theorem 1.}

(Chalco-Cano et al. [17]). Let $\mathrm{x}: \mathrm{T} \rightarrow \mathrm{F}(\mathrm{R})$ be a function and denote 
$[x(t)]^{\alpha}=\left[f_{\alpha}(t), g_{\alpha}(t)\right]$

for each $\alpha \in[0,1]$. Then:

(i) If $\mathrm{x}$ is differentiable in the first form (I), then $f_{\alpha}$ and $g_{\alpha}$ are differentiable functions and

$\left[x^{\prime}(t)\right]^{\alpha}=\left[f_{\alpha}^{\prime}(t), g_{\alpha}^{\prime}(t)\right]$

(ii) If $\mathrm{x}$ is differentiable in the second form (II), then $f_{\alpha}$ and $g_{\alpha}$ are differentiable functions and

$\left[x^{\prime}(t)\right]^{\alpha}=\left[g_{\alpha}^{\prime}(t), f_{\alpha}^{\prime}(t)\right]$

The result of Theorem 1, gives us a procedure to solve the fuzzy initial value problem $X^{\prime}(t)=$ $f(t, X(t))$

$$
X(0)=X_{0},
$$

where $X^{\prime}(t)$ is the generalized derivative (in the first form (I) or second form (II) ) in the sense of Definition 2.7 , see $[17,18]$. In the following result we will show the relationships between the fuzzy solution for problem (11) proposed in Section 3 and the solution of problem (11) when $X(t)$ is the generalized derivative.

The elements $f\left(t_{0}+\mathrm{h}\right), f\left(t_{0}\right)$ at the fight-hand side of Eq. (8), (7) are observed as elements in the Banach space $\mathrm{B}=\bar{C}[0,1] \times \bar{C}[0,1][29]$. Thus if $f\left(t_{0}+\mathrm{h}\right)=(\underline{a}, \bar{a})$ and $f\left(t_{0}\right)=(\underline{b}, \bar{b})$, the difference is simply

$f\left(t_{0}+\mathrm{h}\right)-f\left(t_{0}\right)=(\underline{a}-\underline{b}, \bar{a}-\bar{b})$.

Clearly $\left[f\left(t_{0}+\mathrm{h}\right)-f\left(t_{0}\right)\right] / \mathrm{h}$ may not be a fuzzy number for all h. However, if it approaches $f^{\prime}\left(t_{0}\right)$ in $\mathrm{B}$, and $f^{\prime}\left(t_{0}\right)$ is a fuzzy number, this number is the fuzzy derivative of $\mathrm{f}(\mathrm{t})$ at $\mathrm{t}_{0}$. In this case if $f=(\underline{f}, \bar{f})$ it can be easily shown that

$$
f^{\prime}\left(t_{0}\right)=\left(\underline{f}^{\prime}\left(t_{0}\right), \bar{f}^{\prime}\left(t_{0}\right)\right)
$$

Where $\underline{f}^{\prime}$ and $\bar{f}^{\prime}$ are the classic derivatives of $\underline{f}$ and $\bar{f}$, respectively.

\section{Example 1.}

Consider the fuzzy function

$$
f(t ; \alpha)=\left(\alpha t^{2},\left(2-\alpha^{2}\right) t\right), \quad 0 \leq t \leq 1
$$

for which

$\lim _{\mathrm{h} \rightarrow 0} \frac{f(t+\mathrm{h} ; \alpha)-f\left(t_{0} ; \alpha\right)}{\mathrm{h}}=\left(2 \alpha t_{0},\left(2-\alpha^{2}\right)\right)$ 
is a fuzzy number for t: $0 \leq t \leq \frac{1}{2}$. If $t>\frac{1}{2}$ the right-hand side of Eq. (12) is not a fuzzy number. Consequently, $\mathrm{f}^{\prime}(\mathrm{t})$ does not exist for $\frac{1}{2}<t \leq 1$.

Furthermore, in [25] if $f: R \rightarrow R$ is a real continuous function, then $\hat{f}: F(R) \rightarrow F(R)$ is well-defined function and

$[\hat{f}(U)]^{\alpha}=f\left([U]^{\alpha}\right), \quad \forall \alpha \in[0,1], \quad U \in F(R)$

where $f\left([U]^{\alpha}\right)=\left\{f(x) \mid x \in[U]^{\alpha}\right\}$. Consequently, if $U$ is a fuzzy interval with the closure of it's support is $[\mathrm{U}]^{0}=\left[\mathrm{u}_{1}, \mathrm{u}_{2}\right]$ and $\mathrm{f}$ is a real continuous function, then we have that

$[\hat{f}(U)]^{\alpha}=\left[\min _{x \in\left[u_{1}, u_{2}\right]} f(x), \max _{x \in\left[u_{1}, u_{2}\right]} f(x)\right]$.

Therefore, in order to find the endpoints of (14), we need optimization technique. On the other hand, if $f$ : $\mathrm{R}^{n} \rightarrow \mathrm{R}^{n}$ is a continuous function, then $\hat{f}: F\left(R^{n}\right) \rightarrow F\left(R^{n}\right)$ is a well-defined function, and(see [19])

$$
[\hat{f}(U)]^{\alpha}=f\left([U]^{\alpha}\right), \quad \forall \alpha \in[0,1], \quad \forall U \in F\left(R^{n}\right)
$$

\section{Fuzzy Cauchy problems}

In this section we will give a short review on numerical solutions of fuzzy differential equations. For more detail, refer to [22]. First, we follow Kaleva [21] and define a first-order fuzzy differential equation. We then replace it by its parametric form and solve the new system which consists of two classic ordinary differential equations numerically.

Following [14] we define a first-order fuzzy differential equation by

$x^{\prime}=f(t, x)$

where $\mathrm{x}$ is a fuzzy function of $\mathrm{t}, \mathrm{f}(\mathrm{t}, \mathrm{x})$ - a fuzzy function of the crisp variable $t$ and the fuzzy variable $\mathrm{x}$, and $x^{\prime}$ is the fuzzy derivative of $x[6]$. If an initial value $x\left(t_{0}\right)=x_{0}$ is given, we obtain a fuzzy Cauchy problem of first order:

$x^{\prime}=f(t, x), \quad x\left(t_{0}\right)=X_{0}$.

By Theorem 5.2 in [14] we may replace Eq. (16) by the equivalent system

$\underline{x}^{\prime}(t)=\underline{f}(t, x)=F(t, \underline{x}, \bar{x}), \quad \underline{x}\left(t_{0}\right)=\underline{x_{0}}$,

$\bar{x}^{\prime}(t)=\bar{f}(t, x)=G(t, \underline{x}, \bar{x}), \quad \bar{x}\left(t_{0}\right)=\overline{x_{0}}$

which possesses a unique solution $(\underline{x}, \bar{x}) \in B$ which is a fuzzy function, i.e. for each $t$, the pair $[\underline{x}(t ; \alpha), \bar{x}(t ; \alpha)]$ is a fuzzy number.

The parametric form of Eq. (17) is given by

$$
\begin{array}{ll}
\underline{x^{\prime}}(t ; \alpha)=F(t, \underline{x(t ; \alpha)}, \overline{x(t ; \alpha)}), & \underline{x}\left(t_{0} ; \alpha\right)=\frac{x_{0}(\alpha),}{(18)} \\
\bar{x}^{\prime}(t ; \alpha)=G(t, \underline{x(t ; \alpha), \bar{x}(t ; \alpha)),} & \bar{x}\left(t_{0} ; \alpha\right)=\frac{\overline{x_{0}(\alpha)}}{}
\end{array}
$$

For $\alpha \in[0,1]$ A solution to Eq. (18) must solve Eq. (17) 


\subsection{The fuzzy Euler method}

To integrate the system given in Eq. (18) from to a prefixed $T>t_{0}$, we replace the interval [ $\left.t_{0}, T\right]$ by a set of discrete equally spaced grid points

$t_{0}<t_{1}<t_{2}<\ldots<t_{N}=\mathrm{T}$ at which the exact solution $(\underline{Y}(t ; \alpha), \bar{Y}(t ; \alpha))$ is approximated by some $(\underline{y}(t ; \alpha), \bar{y}(t ; \alpha))$. (Note that throughout each integration,$\alpha$ is unchanged.) The exact and approximate solutions at $t_{n}, 0 \leq n \leq N$ are denoted by $Y_{n}(\alpha)=\left[\underline{Y_{n}}(\alpha), \overline{Y_{n}}(\alpha)\right]$ and $y_{n}(\alpha)=\left[\underline{y_{n}}(\alpha), \overline{y_{n}}(\alpha)\right]$, respectively. The grid points at which the solution is calculated are $t_{n}=t_{0}+n \mathrm{~h}, \quad \mathrm{~h}=\left(\mathrm{T}-\mathrm{t}_{0}\right) / \mathrm{N} ; \quad 1 \leq \mathrm{n} \leq \mathrm{N}$.

The Euler method [13] is based on the first-order approximation of $\underline{Y}^{\prime}(t ; \alpha)$ and $\bar{Y}^{\prime}(t ; \alpha)$ and is given by

$Z^{\prime}(t ; \alpha) \approx \frac{\mathrm{Z}(\mathrm{t}+\mathrm{h} ; \alpha)-\mathrm{Z}(\mathrm{t} ; \alpha)}{\mathrm{h}}$

where $Z(t ; \alpha)$ is $\underline{Y}(t ; \alpha)$ and $\bar{Y}(t ; \alpha)$ alternatively. By virtue of Eq. (20) we obtain

$$
\begin{aligned}
& \underline{Y}_{n+1}(\alpha) \approx \underline{Y}_{n}(\alpha)+h F_{n}(\alpha) \\
& \bar{Y}_{n+1}(\alpha) \approx \bar{Y}_{n}(\alpha)+h G_{n}(\alpha)
\end{aligned}
$$

where

$$
\begin{aligned}
& F_{n}(\alpha) \triangleq F\left[t_{n}, \underline{Y_{n}}(\alpha), \overline{Y_{n}}(\alpha)\right] \\
& G_{n}(\alpha) \triangleq G\left[t_{n}, \underline{Y_{n}}(\alpha), \overline{Y_{n}}(\alpha)\right]
\end{aligned}
$$

Following Eq. (21) we define

$\underline{y}_{n+1}(\alpha)=\underline{y}_{n}(\alpha)+h F\left[t_{n}, \underline{y}_{n}(\alpha), \bar{y}_{n}(\alpha)\right]$

where $\underline{y}_{0}(\alpha) \triangleq \underline{x}_{0}(\alpha), \bar{y}_{0}(\alpha) \triangleq \bar{x}_{0}(\alpha)$. The polygon curves

$$
\begin{gathered}
\underline{y}(t ; h ; \alpha) \triangleq\left\{\left[t_{0}, \underline{y}_{0}(\alpha)\right],\left[t_{1}, \underline{y}_{1}(\alpha)\right], \ldots,\left[t_{N}, \underline{y}_{N}(\alpha)\right]\right\} \\
\bar{y}(t ; h ; \alpha) \triangleq\left\{\left[t_{0}, \bar{y}_{0}(\alpha)\right],\left[t_{1}, \bar{y}_{1}(\alpha)\right], \ldots,\left[t_{N}, \bar{y}_{N}(\alpha)\right]\right\}
\end{gathered}
$$

are the Euler approximates to $\underline{Y}(t ; \alpha)$ and $\bar{Y}(t ; \alpha)$, respectively, over the interval $t_{0} \leq t \leq t_{N}$. The following lemmas will be applied to show convergence of these approximates, i.e.,

$\lim _{h \rightarrow 0} \underline{y}(t ; h ; \alpha)=\underline{Y}(t ; \alpha), \quad \quad \lim _{h \rightarrow 0} \bar{y}(t ; h ; \alpha)=\bar{Y}(t ; \alpha)$.

\section{Lemma 1.}

Let a sequence of numbers $\left\{W_{n}\right\}_{n=0}^{N}$ satisfy

$\left|W_{n+1}\right| \leq A\left|W_{n}\right|+B, \quad 0 \leq n \leq N-1$

for some given positive constants $A$ and $B$. Then

$\left|W_{n}\right| \leq A^{N}\left|W_{0}\right|+B \frac{A^{n}-1}{A-1}, \quad 0 \leq n \leq N-1$

The proof, using mathematical induction is straightforward.

\section{Lemma 2.}

Let the sequences of numbers $\left\{W_{n}\right\}_{n=0}^{N},\left\{V_{n}\right\}_{n=0}^{N}$ satisfy

$\left|W_{n+1}\right| \leq\left|W_{n}\right|+A \max \left\{\left|W_{n}\right|,\left|V_{n}\right|\right\}+B$ 
$\left|V_{n+1}\right| \leq\left|V_{n}\right|+A \max \left\{\left|V_{n}\right|,\left|W_{n}\right|\right\}+B$

for some given positive constants $A$ and $B$, and denote

Then

$$
U_{n}=\left|W_{n}\right|+\left|V_{n}\right|, \quad 0 \leq n \leq N
$$

where $\bar{A}=1+2 A$ and $\bar{B}=2 B$.

$$
U_{n} \leq \bar{A}^{n} U_{0}+\bar{B} \frac{\bar{A}^{n}-1}{\bar{A}-1}, \quad 0 \leq n \leq N
$$

Our next result determines the point wise convergence of the Euler approximates to the exact solution. Let $F(t, u, v)$ and $G(t, u, v)$ be the functions $\mathrm{F}$ and $\mathrm{G}$ of Eq. (17) where $\mathrm{u}$ and $\mathrm{v}$ are constants and $u \leq v$. In other words $F(t, u, v)$ and $G(t, u, v)$ are obtained by substituting $\mathrm{x}=(\mathrm{u}, \mathrm{v})$ in Eq. (17). The domain where $\mathrm{F}$ and $\mathrm{G}$ are defined is therefore

$K=(t, u, v) \mid t_{0} \leq t \leq T,-\infty<v<\infty,-\infty<u \leq v$.

\section{Theorem 2}

For arbitrary fixed : $0 \leq \alpha \leq 1$, the two step approximates of Eq.(24) converge to the exact solution $[Y(t, \alpha), \overline{Y(t, \alpha)]}$.

\section{Zadeh's extension principle}

In [26], Zadeh proposed the so-called extension principle which becomes an important tool in fuzzy set theory and its applications. The principal idea of Zadeh's extension principle is that each function $f: X \rightarrow Y$ induces another function $\hat{f}: F(X) \rightarrow F(Y)$ defined for each fuzzy interval $\mathrm{U}$ in $\mathrm{X}$ by

$\hat{f}(U)(y)= \begin{cases}\sup _{x \in f^{-1}(y)} U(x) & \text { if } y \in \operatorname{range}(f) \\ 0 & \text { if } y \notin \operatorname{range}(f)\end{cases}$

In this case, the function $\hat{f}$ is said to be obtained from $\mathrm{f}$ by Zadeh's extension principle. In general, the computation of $\hat{f}$ is not an easy task. An exception occurs when $\mathrm{f}$ is monotone.

The fuzzy initial value problem solving by this method:

$X^{\prime}(t)=f(t, X(t)), X(0)=X 0$,

where $f:[0, T] \times \mathrm{F}(U) \rightarrow \mathrm{F}\left(\mathrm{R}^{\mathrm{n}}\right)$ is obtained by Zadeh's extension principle from a continuous function $g:[0, T] \times U \rightarrow \mathrm{R}^{\mathrm{n}}$, where $U \subset \mathrm{R}^{\mathrm{n}}$. Note that $f$ is continuous because $g$ is continuous (see [19]) and by(13) we have

$[f(t, X)]^{\alpha}=g\left(t,[X]^{\alpha}\right)$

, where $g(t, A)=\{g(t, a) \mid a \in A\}$.

Associated with $\mathrm{FDE}(30)$ we can consider the deterministic differential equation (DDE):

$x^{\prime}(t)=g(t, x(t)), \quad x(0)=c$

where $x^{\prime}(t)$ is the derivative (crisp) of a function $x:[0, T] \rightarrow \mathrm{R}^{\mathrm{n}}$.

We obtain a fuzzy solution for (30) derived from (32) suppose that problem (32) has the solution $x(t, c)$. Then, applying the Zadeh's extension principle to $x(t, c)$ in relation to the parameter $c$, we obtain the extension $X(t)=\hat{x}\left(t, X_{0}\right)$, for each $t$ fixed, which is a fuzzy solution of problem (30)(in the sequel, this extended solution will be called the fuzzy solution for problem (30)).

Theorem3. 
Let $U$ be an open set in $R^{n}$ and $\left[X_{0}\right]^{\alpha} \subset U$. Suppose that $g$ is continuous and that for each $c \in U$ there exists a unique solution $x(\cdot, c)$ of the deterministic problem (32) and that $x(t, \cdot)$ is continuous on $U$ for eac $t \in$ $[0, T] h$ fixed. Then, there exists a unique fuzzy solution $X(t)=\hat{x}\left(t, X_{0}\right)$ of the FDE (30).

\section{Example}

Consider the initial value problem

$$
\left\{\begin{array}{c}
y^{\prime}(t)=-y(t) \\
c=y(0)=(0.96+0.04 \alpha, 1.01-0.01 \alpha)
\end{array}\right.
$$

The problem (a) has the exact solution $y(t, c)=c e^{-t}$.

Note that $x(t, c)$ is continuous in $c \in \mathrm{R}$ for each $t \geq 0$ fixed

\section{1.solving by Euler method}

$y(t)=\left((0.96+0.04 \alpha) e^{-t},(1.01-0.01 \alpha) e^{-t}\right)$

$y(0.1)=\left((0.96+0.04 \alpha) e^{-0.1},(1.01-0.01 \alpha) e^{-0.1}\right)$

The exact solution at $t=1$ is given by

$Y(1, \alpha)=\left((0.96+0.04 \alpha) e^{-1},(1.01-0.01 \alpha) e^{-1}\right) \quad 0 \leq \alpha \leq 1$

Using the Euler approximation with $N=10$ we obtain

Table 1. the numerical results illustrated by applying Euler method

\begin{tabular}{|c|c|c|c|c|c|c|}
\hline$\alpha$ & $y$ & $\underline{Y}$ & ERROR & $\bar{y}$ & $\bar{Y}$ \\
\hline 0 & .2527616437 & .3531642636 & -.1004026199 & .4748452781 & .3715582356 & .1032870425 \\
\hline 0.1 & .2644198408 & .3546357813 & $-0.902159405 \mathrm{e}-1$ & .4642951118 & .3711903562 & $0.931047556 \mathrm{e}-1$ \\
\hline 0.2 & .2760780380 & .3561072991 & $-0.800292611 \mathrm{e}-1$ & .4537449455 & .3708224767 & $0.829224688 \mathrm{e}-1$ \\
\hline 0.3 & .2877362351 & .3575788168 & $-0.698425817 \mathrm{e}-1$ & .4431947792 & .3704545973 & $0.727401819 \mathrm{e}-1$ \\
\hline 0.4 & .2993944323 & .3590503346 & $-0.596559023 \mathrm{e}-1$ & .4326446129 & .3700867178 & $0.625578951 \mathrm{e}-1$ \\
\hline 0.5 & .3110526294 & .3605218524 & $-0.494692230 \mathrm{e}-1$ & .4220944466 & .3697188384 & $0.523756082 \mathrm{e}-1$ \\
\hline 0.6 & .3227108265 & .3619933701 & $-0.392825436 \mathrm{e}-1$ & .4115442803 & .3693509590 & $0.421933213 \mathrm{e}-1$ \\
\hline 0.7 & .3343690237 & .3634648879 & $-0.290958642 \mathrm{e}-1$ & .4009941140 & .3689830795 & $0.320110345 \mathrm{e}-1$ \\
\hline 0.8 & .3460272208 & .3649364057 & $-0.189091849 \mathrm{e}-1$ & .3904439477 & .3686152001 & $0.218287476 \mathrm{e}-1$ \\
\hline 0.9 & .3576854180 & .3664079234 & $-0.87225054 \mathrm{e}-2$ & .3798937814 & .3682473206 & $0.116464608 \mathrm{e}-1$ \\
\hline 1 & .3693436151 & .3678794412 & $0.14641739 \mathrm{e}-2$ & .3693436151 & .3678794412 & $0.14641739 \mathrm{e}-2$ \\
\hline
\end{tabular}

\subsection{Solving by Zadeh's extension principle}

Note that the initial condition $C$ is any fuzzy interval .We apply the Zadeh's extension principle to $x(t, c)$ in relation to $c$, for each $t \geq 0$ fixed. Then we obtain the unique fuzzy solution $X(t)=\hat{x}(t, C)$ of problem (a) for any initial condition $C$, with $C$ a fuzzy interval, which is given by $X(t)=C \cdot \mathrm{e}^{-\mathrm{t}}, \quad t \geq 0$, where $(\cdot)$ is the scalar multiplication (1): 


$$
\begin{aligned}
& \left(\begin{array}{cc}
0 & x<0.96
\end{array}\right. \\
& C(x)=\left\{\begin{array}{cc}
\frac{x-1.01}{0.04} & 0.96 \leq x \leq 1 \\
\frac{1.01-x}{0.01} & 1 \leq x \leq 1.01 \\
0 &
\end{array}\right. \\
& \begin{array}{rrr}
0 & x>1.01 \\
0 & & y<e^{-1.01}
\end{array} \\
& \hat{f}(C)(y)= \begin{cases}\frac{\text { Lny-1.01 }}{0.01} & e^{-1.01} \leq y \leq e^{-1} \\
\frac{1.01+\text { Lny }}{0.04} & e^{-1} \leq y \leq e^{-0.96} \\
0 & \end{cases} \\
& X(0.1)=\left\{\begin{array}{cc}
0 & y<e^{-1.11} \\
\frac{L n y-1.01 \cdot e^{-0.1}}{0.01 . e^{-0.1}} & e^{-1.11} \leq y \leq e^{-1.1} \\
1.01 . e^{-0.1}+\operatorname{Lny} &
\end{array}\right. \\
& \frac{1.01 . e^{-0.1}+\text { Lny }}{0.04 e^{-0.1}} \quad e^{-1.1} \leq y \leq e^{-1.06} \\
& {[X(t)]^{\alpha}=\left(\begin{array}{cc}
0 & y>e^{-1.06} \\
-1.11 & +\alpha\left(e^{-1.1}-e^{-1.11}\right), e^{-1.06}-\alpha\left(e^{-1.06}-e^{-1.1}\right)
\end{array}\right)}
\end{aligned}
$$

Table 2. the numerical results illustrated by applying Zadeh's extension principle

\begin{tabular}{|c|c|c|c|c|c|c|}
\hline$\alpha$ & $\underline{y}$ & $\underline{Y}$ & ERROR & $\bar{y}$ & $\bar{Y}$ & ERROR \\
\hline 0 & 0.3295589611 & 0.3531642636 & -0.023605302524811 & 0.3464558103 & 0.3715582356 & -0.025102425269943 \\
\hline 0.1 & 0.3298901733 & 0.3546357813 & -0.024745607951792 & 0.3450973376 & 0.3711903562 & -0.026093018475126 \\
\hline 0.2 & 0.3302213856 & 0.3561072991 & -0.025885913454189 & 0.3437388650 & 0.3708224767 & -0.027083611697152 \\
\hline 0.3 & 0.3305525979 & 0.3575788168 & -0.027026218956586 & 0.3423803923 & 0.3704545973 & -0.028074204919178 \\
\hline 0.4 & 0.3308838101 & 0.3590503346 & -0.028166524458982 & 0.3410219197 & 0.3700867178 & -0.029064798141205 \\
\hline 0.5 & 0.3312150224 & 0.3605218524 & -0.029306829961379 & 0.3396634470 & 0.3697188384 & -0.030055391363231 \\
\hline 0.6 & 0.3315462346 & 0.3619933701 & -0.030447135463776 & 0.3383049744 & 0.3693509590 & -0.031045984585257 \\
\hline 0.7 & 0.3318774469 & 0.3634648879 & -0.031587440966173 & 0.3369465017 & 0.3689830795 & -0.032036577807284 \\
\hline 0.8 & 0.3322086592 & 0.3649364057 & -0.032727746468569 & 0.3355880290 & 0.3686152001 & -0.033027171029310 \\
\hline 0.9 & 0.3325398714 & 0.3664079234 & -0.033868051970966 & 0.3342295564 & 0.3682473206 & -0.034017764251337 \\
\hline 1 & 0.3328710837 & 0.3678794412 & -0.035008357473363 & 0.3328710837 & 0.3678794412 & -0.035008357473363 \\
\hline
\end{tabular}




\section{Green:Exact solution}

\section{Blue:Euler method}

\section{Red: Zadeh's extension principle}

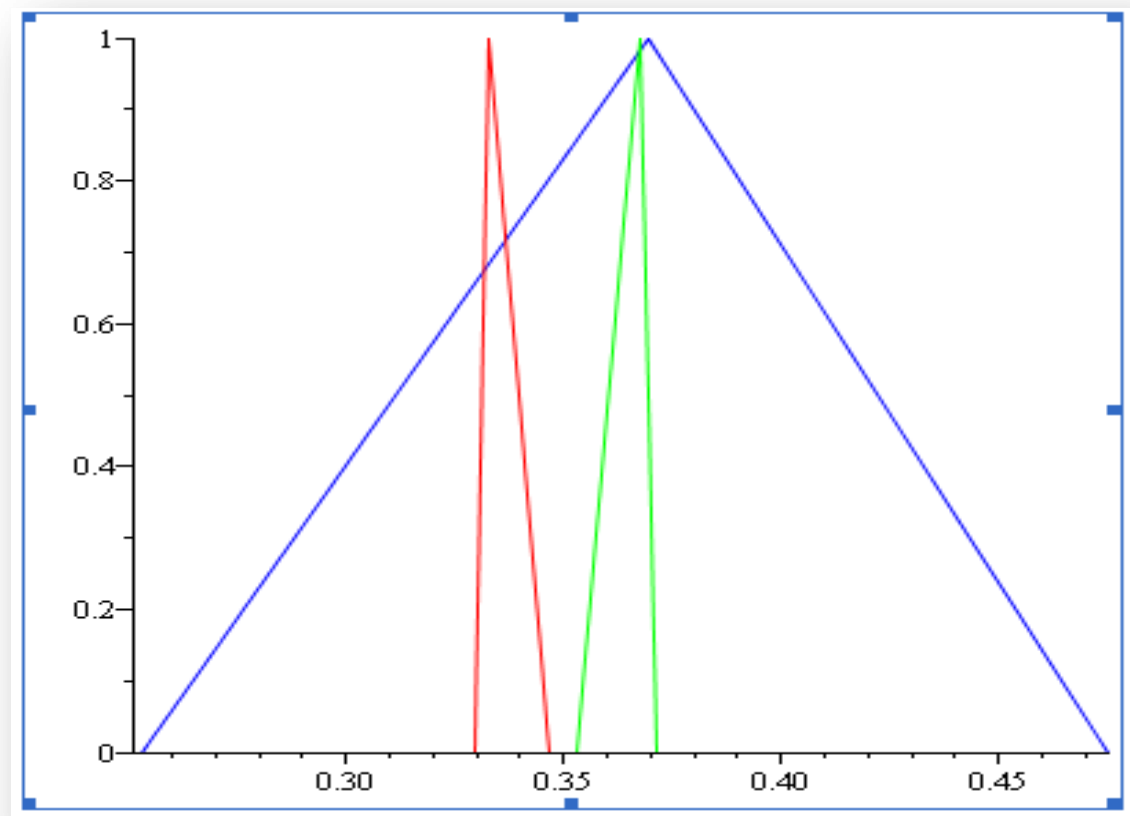

Figure 2: the comparison of two numerical methods with the exact solution

\section{Conclusion}

In this paper two numerical algorithms for solving fuzzy ordinary differential equations is considered. A scheme based on two different methods is discussed, Zadeh's extension principle and Euler method. By Euler method we can approximate fuzzy ? -reachable set, since $[X(t)]^{\alpha}$ is an interval. Numerical example shows the efficiency of implemented numerical method. Also We obtain a fuzzy solution for this class of FDE by applying the Zadeh's extension principle to the deterministic solution associated with the fuzzy problem.

we study fuzzy differential equations (FDE) and We give some properties and we show that The method presented in this paper greatly improve the solution technique for the computation of functions that take fuzzy sets as their arguments.

\section{References}

[1] Ming Ma , Menahem Friedman , Abraham Kandel , Numerical solutions of fuzzy differential equations , Fuzzy Sets and Systems 105 (1999) 133-138. 
[2] Y.Chalco-Canoa, H.Roman-Flore , Comparation between some approaches to solve fuzzy differential equations , Fuzzy Sets and Systems 160 (2009) 1517-1527.

[3] Menahem Friedman, Ming Ma, Abraham Kandel , Numerical solutions of fuzzy differential and integral equations Fuzzy Sets and Systems 106 (1999) 35-48.

[4] M. Z. Ahmad and 2M. K. Hasan , A New Approach for Computing Zadeh's Extension Principle , MATEMATIKA, 2010, Volume 26, Number 1, 71-81.

[5] Ch.Carlsson , R.Fuller , P.Majlender , An extension principle for interactive fuzzy numbers Fuzzy Sets and Systems (submitted).

[6] M.L. Puri, D.A. Ralescu, Differentials of fuzzy functions, J. Math. Anal. Appl. 91 (1983) 552-558.

[7] O.Solaymani Fard , A Numerical Scheme for Fuzzy Cauchy Problems, Journal of Uncertain Systems Vol.3, No.4, pp.307-314, 2009.

[8] E. Babolian,S. Abbasbandy,M. Alavi , Numerical solution of fuzzy differential inclusion by Euler method, J. Sci. I . A . U (JSIAU),Vol 18, No . 70.2, Winter 2009.

[9] D. N. GEORGIOU and I. E. KOUGIAS , ON CAUCHY PROBLEMS FOR FUZZY DIFFERENTIAL EQUATIONS , IJMMS 2004:15, 799-805.

[10] A. Khastan, F. Bahrami, K. Ivaz , SOLVING HIGHER-ORDER FUZZY DIFFERENTIALEQUATIONS UNDER GENERALIZED

DIFFERENTIABILITY, ROMAI J, 5, 1(2009), 85-87.

[11] T. Allahviranloo 1, N. Ahmady, E. Ahmady ,Two Step Method for

Fuzzy Differential Equations , International Mathematical Forum, 1, 2006, no. 17, 823-832.

[12] P. Diamond, P. Kloeden, Metric spaces of fuzzy sets, Fuzzy Sets and Systems 35 (1990) 241-249.

[13] M. Friedman, A. Kandel, Fundamentals of Computer Numerical Analysis, CRC Press, Boca Raton, FL (1994), pp. 390-400, 441-444.

[14] O. Kaleva, Fuzzy differential equations, Fuzzy Sets and Systems 24 (1987) 301-317.

[15] E.P. Klement, M.L. Puri, D.A. Ralescu, Limit theorems for fuzzy random variables, Proc. Roy. Soc. London Ser. A. 407 (1986) 171-182.

[16] Wu Congxin, Ma Ming, On embedding problem of fuzzy number spaces. Part 1, Fuzzy Sets and Systems 44 (1991) 33 -38.

[17] Y. Chalco-Cano, H. Román-Flores, On the new solution of fuzzy differential equations, Chaos Solitons Fractals 38 (2006) 112-119.

[18] O. Kaleva, A note on fuzzy differential equations, Nonlinear Anal. 64 (2006) 895-900.

[19] H. Roman-Flores, L. Barros, R. Bassanezi, A note on the Zadeh's extensions, Fuzzy Sets and Systems 117 (2001) 327-331.

[20] R. Goetscbel, W. Voxman, Elementary calculus, Fuzzy Sets and Systems 18 (1986) 31-43.

[21] O. Kaleva, Fuzzy differential equations, Fuzzy Sets and Systems 24 (1987) 301-317.

[22] Ma Ming, M. Friedman, A. Kandel, Numerical solution of fuzzy differential equations, Fuzzy Sets and Systems, to appear.

[23] M.L. Purl, D. Ralescu, Differential for fuzzy function, J. Math. Anal. Appl. 91 (1983) 552-558.

[24] M.L. Purl, D. Ralescu, Fuzzy random variables, J. Math. Anal. Appl. 114 (1986) 409-422.

[25] H. Rom'an-Flores, L. Barros, and R. Bassanezi. A note on Zadeh's extension principle. Fuzzy Sets and Systems, 117:327-331, 2001.

[26] L. A. Zadeh. Fuzzy sets. Information and Control, 8:338-353, 1965.

[27] S. S. L. Chang and L. A. Zadeh, On fuzzy mapping and control, IEEE Trans. Systems Man Cybernet. 2 (1972), 30-34.

[28] Towards fuzzy differential calculus. III. Differentiation, Fuzzy Sets and Systems 8 (1982), no. 3, 225233.

[29] Wu Congxin, Ma Ming, On embedding problem of fuzzy number spaces, Fuzzy Sets and Systems 44 (1991) 33-38;45 (1992) 189-202; 46 (1992) 281-286. 